\title{
Biología trófica de los juveniles del género Liza (Pisces: Mugilidae) en la laguna costera del Mar Menor (SE Península Ibérica)
}

\author{
David Verdiell-Cubedo*, Andrés Egea-Serrano, Francisco J. Oliva-Paterna \& Mar Torralva \\ Departamento de Zoología y Antropología Física, Facultad de Biología. Universidad de Murcia, 30100 Murcia, \\ España.
}

* Autor responsable de la correspondencia: verdiell@um.es.

\section{RESUMEN}

Biología trófica de los juveniles del género Liza (Pisces: Mugilidae) en la laguna costera del Mar Menor (SE Península Ibérica)

En el presente trabajo se presentan datos sobre la alimentación de juveniles de tres especies de mugílidos presentes en la laguna costera del Mar Menor: Liza saliens (Risso, 1810), L. aurata (Risso, 1810) y L. ramado (Risso, 1810). La dieta fue omnívora para las tres especies, consumiendo una gran variedad de presas, tanto de origen animal como vegetal. Se encontraron diferencias interespecíficas en la composición de la misma, así como diferencias intraespecíficas en función de la campaña de muestreo. Además, se observaron cambios en la dieta en función de la talla de los ejemplares, situación probablemente relacionada con cambios ontogénicos en la dieta de las especies estudiadas.

Palabras clave: Liza spp., alimentación, juveniles, laguna costera, Mar Menor.

\begin{abstract}
Trophic biology of juvenile stages of Liza species (Pisces: Mugilidae) in the Mar Menor coastal lagoon (SE Iberian Peninsula)

In this work data is presented concerning the food and feeding habits of three species of mullet juveniles that are present in the Mar Menor coastal lagoon: Liza saliens (Risso, 1810), L. aurata (Risso, 1810) and L. ramado (Risso, 1810). Diet of of the three species was omnivorous; they fed on numerous prey items, both animal and vegetal preys. Interspecific differences on diet composition were found, as well as intraspecific differences from sampling periods. Moreover, size-related differences in their feeding habits were found, suggesting the existence of ontogenic changes in the diet of the studied species.
\end{abstract}

Keywords: Liza spp., feeding habits, juvenile fishes, coastal lagoon, Mar Menor.

\section{INTRODUCCIÓN}

La familia Mugilidae se caracteriza por presentar ciclos de vida anfídromos, realizando migraciones de cierta importancia, con fines no reproductivos, hacia los estuarios y lagunas costeras (Granado-Lorencio, 1996). En dichos sistemas acuáticos suele presentarse como una de las familias dominantes dentro de la comunidad íctica (Strydom, 2003; Koutrakis et al., 2005). La plasticidad ecológica que presenta dicha familia se traduce, entre otros, en la capacidad de consumir una gran variedad de alimentos: detritos, algas, crustáceos, moluscos, insectos, etc. (FernándezDelgado et al., 2000; Cardona, 2001). En este sentido, contribuye de manera decisiva en el flujo de materia y energía en dichos sistemas (Laffaille et al., 2002; Almeida, 2003).

A su vez, las especies de esta familia son objeto de importantes pesquerías y utilizadas en piscicultura extensiva y semiintensiva (Ferrari \& Chieregato, 1981; Arias \& Drake, 1990; Cardona \& Castelló, 1993; Hotos \& Vlahos, 1998). En el Mar Menor las pesquerías de mu- 
gílidos se realizan principalmente mediante artes fijas de pesca $y$, aunque en la actualidad sus capturas se han visto mermadas, todavía presentan importancia comercial (Pérez-Ruzafa \& Marcos, 2003).

En la Península Ibérica, la alimentación de los estados juveniles de mugílidos ha sido tratada únicamente en el delta del Ebro (Gisbert et al., 1996), en las marismas de la bahía de Cádiz (Arias \& Drake, 1990) y en el estuario del Guadalquivir (Baldó \& Drake, 2002), si bien en estos estudios no se analiza el patrón estacional de alimentación.

El objetivo de este trabajo, que constituye la primera aportación al conocimiento de los hábitos alimentarios de los juveniles de mugílidos en el Mar Menor y, por tanto, en el sureste peninsular, es determinar la dieta de los estados juveniles de las especies del género Liza presentes en dicha laguna: Liza saliens (Risso, 1810), L. aurata (Risso, 1810) y L. ramado (Risso, 1810). A su vez, se analiza la existencia de variaciones inter e intraespecíficas en la dieta de los estados juveniles de estas especies.

\section{ÁREA DE ESTUDIO}

El Mar Menor es una de las lagunas costeras de mayor extensión e importancia de la cuenca mediterránea, con una superficie aproximada de $135 \mathrm{~km}^{2}$, y localizada en el SE de la Península Ibérica. La laguna muestra un rango de salinidad que oscila entre los 39 y $45 \%$, y un rango de temperatura de sus aguas entre 10 y $30{ }^{\circ} \mathrm{C}$. Sus fondos están recubiertos principalmente por una densa pradera de la macroalga Caulerpa prolifera, aunque en las zonas mas someras existen praderas de la fanerógama Cymodocea nodosa y praderas mixtas de $C$. nodosa y $C$. prolifera (Pérez-Ruzafa \& Marcos, 2003).

Durante las últimas décadas, la laguna ha sufrido un aumento de las actividades relacionadas con el turismo y la contaminación orgánica e inorgánica de sus aguas producto de la entrada de nutrientes procedentes del Campo de Cartagena, área limítrofe a la laguna de gran importancia agrícola. Todo ello ha provocado una modifi- cación drástica de las condiciones del hábitat en el Mar Menor (Pérez-Ruzafa \& Marcos, 2003).

Finalmente, la laguna mantiene importantes pesquerías de peces (Anguilla anguilla, Atherina boyeri, Sparus aurata, Mugil cephalus, Liza spp. y Dicentrarchus labrax) (Pérez-Ruzafa \& Marcos, 2003; Andreu-Soler et al., 2003).

Tabla 1. Especies de mugílidos estudiadas ( $\mathrm{n}=$ número de ejemplares, Ls = longitud estándar media). Mullet species studied ( $n=$ number of fish, $L s=$ mean standard length).

\begin{tabular}{lcccc}
\hline Especie & Estación & $n$ & $\mathrm{Ls}$ & $\mathrm{Ls}_{\min }-\mathrm{Ls}_{\max }$ \\
\hline Liza saliens & verano & 42 & 3.9 & $2.2-6.8$ \\
& otoño & 40 & 2.9 & $1.9-4.3$ \\
& invierno & 45 & 2.8 & $1.9-4.1$ \\
& primavera & 40 & 3.2 & $2.1-4.7$ \\
Liza aurata & verano & 45 & 4.5 & $3.3-6.3$ \\
& otoño & 43 & 2.2 & $1.8-2.7$ \\
& invierno & 42 & 2.7 & $1.8-3.9$ \\
& primavera & 40 & 3.3 & $2.5-4.7$ \\
Liza ramado & verano & 52 & 3.4 & $1.8-8.5$ \\
& otoño & - & - & - \\
& invierno & 40 & 1.9 & $1.6-2.0$ \\
& primavera & 49 & 2.7 & $1.9-7.7$ \\
\hline
\end{tabular}

\section{MATERIAL Y MÉTODOS}

Los ejemplares utilizados para el presente estudio fueron capturados en las zonas someras de la laguna (profundidad $<1 \mathrm{~m}$ ), durante cuatro campañas de muestreo: Julio de 2003 (verano), Noviembre de 2003 (otoño), Febrero de 2004 (invierno) y Abril de 2004 (primavera). Las capturas se realizaron mediante una red de arrastre manual de $10 \mathrm{~m}$ de longitud y $0.5 \mathrm{~mm}$ de luz de malla, y los ejemplares se conservaron a $-15{ }^{\circ} \mathrm{C}$ hasta el momento de su estudio. En el laboratorio se procedió a su identificación sensu Arias \& Drake (1990) y a la determinación individualizada de la longitud estándar (Ls $\pm 1 \mathrm{~mm})$ (Tabla 1).

El contenido estomacal de 167 individuos de L. saliens, 170 de $L$. aurata y 141 de $L$. ramado fue estudiado bajo microscopio óptico (40 y 100 aumentos, Leika Galen III $\left.{ }^{\circledR}\right)$. Las presas de origen animal se determinaron hasta el nivel de grandes grupos taxonómicos: insectos, crustáceos, nemátodos, poliquetos y huevos sin 
identificar. El resto de presas se clasificaron en: detritus, algas filamentosas, microalgas y arena.

La caracterización de la dieta se realizó registrando la relación de presas presentes en cada individuo. La frecuencia de ocurrencia $(\mathrm{Fi})$ de cada presa ingerida se calculó con la expresión (Drake \& Arias, 1984):

$$
F_{i}=\left(N_{i} / N_{s}\right) \times 100
$$

donde $N_{i}$ es el número de estómagos donde aparece la presa $i$ y $N_{s}$ el número total de estómagos examinados para la especie $s$. El cálculo de $F_{i}$ se realizó para el total de ejemplares de cada especie a lo largo del periodo de estudio y para el total de ejemplares de cada especie en cada campaña de muestreo.

Se analizaron las diferencias interespecíficas en el consumo de presas para todo el periodo de estudio así como las diferencias intraespecíficas en función de la campaña de muestreo. Dichas diferencias se comprobaron mediante la prueba de la $\chi^{2}$.

Además, se analizaron las correlaciones existentes entre la Ls de los estados juveniles del género Liza y la presencia de las distintas presas, así como la correlación entre la presencia de las mismas, mediante el análisis de correlación de Spearman.

Los análisis estadísticos fueron llevados a cabo con el paquete estadístico SPSS ${ }^{\circledR}$, aceptándose un nivel de significación del $95 \%$.

\section{RESULTADOS}

Durante el periodo de estudio L. saliens mostró una dieta basada principalmente en el consumo de crustáceos $(48.5 \%)$ y detritus $(46.1 \%)$, esta especie complementó su dieta con huevos $(27.6 \%)$. Del mismo modo, la dieta de $L$. aurata estuvo dominada por la presencia de crustáceos $(49.4 \%)$ y detritus (30.6\%), aunque sus presas secundarias fueron insectos y huevos $(21.2 \%)$. Los ejemplares de $L$. ramado consumieron principalmente crustáceos y huevos $(41.1 \%)$, siendo las microalgas la presa secundaria $(40.4 \%)$ (Tabla 2$)$.

No se encontró dependencia entre la presencia
Tabla 2. Frecuencia de aparición $\left(F_{i}\right)$ de las presas halladas en los estómagos de los estados juveniles de las especies del género Liza, en la laguna costera del Mar Menor. Occurrence frequency $\left(F_{i}\right)$ of preys found in the stomach of juvenile stages of Liza species captured in the Mar Menor coastal lagoon.

\begin{tabular}{cccc}
\hline & Liza saliens & Liza aurata & Liza ramado \\
\hline Detritus & 46.1 & 30.6 & 27.0 \\
Algas filamentosas & 7.2 & 1.2 & 1.4 \\
Microalgas & 1.2 & 2.4 & 40.4 \\
Arena & 27.6 & 34.1 & 24.1 \\
Insectos & 5.4 & 21.2 & 4.3 \\
Crustáceos & 48.5 & 49.4 & 41.1 \\
Nemátodos & 1.8 & 5.3 & 9.9 \\
Poliquetos & 0.0 & 2.4 & 0.0 \\
Huevos & 27.6 & 21.2 & 41.1 \\
\hline
\end{tabular}

de crustáceos $\left(\chi^{2}=2.475 ;\right.$ g.l. $\left.=2 ; \mathrm{P}=0.290\right)$ y $\operatorname{arena}\left(\chi^{2}=3.976 ;\right.$ g.l. $\left.=2 ; \mathrm{P}=0.137\right)$ en función de la especie. Sin embargo, sí se obtuvo dependencia significativa en la ocurrencia del resto de presas: detritus $\left(\chi^{2}=14.539\right.$; g.1. $=2$; $\mathrm{P}=0.001)$, algas filamentosas $\left(\chi^{2}=11.703\right.$; g.l. $=2 ; \mathrm{P}=0.003)$, microalgas $\left(\chi^{2}=129.839 ;\right.$ g.l. $=2 ; \mathrm{P}=0.001)$, insectos $\left(\chi^{2}=30.662 ;\right.$ g.l. $=2$; $\mathrm{P}=0.001)$, nemátodos $\left(\chi^{2}=9.842 ;\right.$ g.l. $=2 ; \mathrm{P}=$ $0.007)$, poliquetos $\left(\chi^{2}=7.308 ;\right.$ g.1. $\left.=2 ; \mathrm{P}=0.026\right)$ y huevos $\left(\chi^{2}=15.201 ;\right.$ g.l. $\left.=2 ; \mathrm{P}=0.001\right)$.

A nivel intraespecífico se observan diferencias en la composición de la dieta en función de la campaña (Tabla 3). Así, L. saliens mostró durante el verano una dieta basada principalmente en los detritus $(64.3 \%)$ y los crustáceos $(21.4 \%)$; durante la campaña de otoño la dieta estuvo formada principalmente por crustáceos $(60 \%)$ y en segundo lugar por detritus (45\%); durante el invierno sus presas preferenciales fueron los crustáceos y los huevos (51.1\%), seguido por los detritus (42.2\%); y finalmente, en la campaña de primavera, los crustáceos $(62.5 \%)$ y los detritus $(32.5 \%)$ fueron las presas mas consumidas. L. aurata mostró, durante la campaña de verano, preferencia por los detritus $(57.8 \%)$, los crustáceos $(24.4 \%)$ y los insectos $(20.0 \%)$; durante la campaña de otoño los crustáceos y los huevos fueron sus presas principales $(76.7 \% \mathrm{y}$ $46,5 \%$ respectivamente); durante la campaña de invierno fueron los crustáceos $(54.8 \%)$, junto con los nemátodos y huevos (19.1\%); y, durante 
Tabla 3. Frecuencia de aparición estacional de las presas $\left(F_{i}\right)$ halladas en los estómagos de los estados juveniles de las especies del género Liza, en la laguna costera del Mar Menor. Seasonal occurrence frequency of preys $\left(F_{i}\right)$ found in the stomach of juvenile stages of Liza species captured in the Mar Menor coastal lagoon.

\begin{tabular}{|c|c|c|c|c|c|c|c|c|c|c|c|c|}
\hline & \multicolumn{4}{|c|}{ Liza saliens } & \multicolumn{4}{|c|}{ Liza aurata } & \multicolumn{4}{|c|}{ Liza ramado } \\
\hline & verano & otoño & invierno & primavera & verano & otoño & invierno & primavera & verano & otoño & invierno & primavera \\
\hline Detritus & 64.3 & 45.0 & 42.2 & 32.5 & 57.8 & 11.6 & 14.3 & 37.5 & 44.2 & - & 7.5 & 27.9 \\
\hline Algas filamentosas & 9.5 & 12.5 & 0.0 & 7.5 & 0.0 & 2.3 & 0.0 & 2.5 & 1.9 & - & 0.0 & 2.3 \\
\hline Microalgas & 0.0 & 0.0 & 0.0 & 5.0 & 0.0 & 0.0 & 9.5 & 0.0 & 50.0 & - & 22.5 & 39.5 \\
\hline Arena & 40.5 & 22.5 & 28.9 & 17.5 & 75.6 & 0.0 & 35.7 & 22.5 & 32.7 & - & 5.0 & 27.9 \\
\hline Insectos & 11.9 & 2.5 & 0.0 & 7.5 & 20.0 & 14.0 & 16.7 & 35.0 & 3.9 & - & 0.0 & 9.3 \\
\hline Crustáceos & 21.4 & 60.0 & 51.1 & 62.5 & 24.4 & 76.7 & 54.8 & 42.5 & 26.9 & - & 75.0 & 32.6 \\
\hline Nemátodos & 0.0 & 2.5 & 4.4 & 0.0 & 0.0 & 0.0 & 19.1 & 2.5 & 3.9 & - & 17.5 & 7.0 \\
\hline Poliquetos & 0.0 & 0.0 & 0.0 & 0.0 & 0.0 & 0.0 & 9.5 & 0.0 & 0.0 & - & 0.0 & 0.0 \\
\hline Huevos & 9.5 & 22.5 & 51.1 & 25.0 & 4.4 & 46.5 & 19.1 & 15.0 & 13.5 & - & 75.0 & 41.9 \\
\hline
\end{tabular}

la primavera, su dieta se basó principalmente en crustáceos $(42.5 \%)$, detritus $(37.5 \%)$ e insectos $(35.0 \%)$. Durante el verano, $L$. ramado presentó una dieta basada principalmente en el consumo de microalgas $(50.0 \%)$, detritus $(44.2 \%)$ y crustáceos $(26.9 \%)$; durante la campaña de invierno la dieta estuvo formada casi exclusivamente por crustáceos y huevos (75.0\%), aunque el $22.5 \%$ de los individuos consumió microalgas; finalmente, en la campaña de primavera, los huevos (41.9\%), las microalgas $(39.5 \%$ ) y los crustáceos $(32.6 \%)$, fueron las presas principales.

La prueba de la $\chi^{2}$ mostró dependencia significativa entre el consumo de las distintas presas por especie y las campañas de muestreo
(Tabla 4). Así, L. saliens mostró dependencia significativa estacional en el consumo de detritus, crustáceos y huevos. L. aurata mostró dependencia significativa estacional en el consumo de detritus, microalgas, arena, crustáceos, nemátodos, poliquetos y huevos. Por último, $L$. ramado presentó dependencia significativa estacional en el consumo de detritus, microalgas, arena, crustáceos y huevos.

Los resultados del análisis de correlación de Spearman se muestran en la Tabla 5. Con respecto a las correlaciones entre la Ls de los estados juveniles y las presas, cabe destacar la relación positiva entre la Ls y la presencia de detritus y arena en los estómagos, así como la

Tabla 4. Resultados del análisis de independencia (prueba de la $\chi^{2}$ ) entre el consumo de las distintas presas por especie y las campañas de muestreo. Results of independence analysis $\left(\chi^{2}\right.$ test $)$ between prey occurrence and sampling periods.

\begin{tabular}{|c|c|c|c|c|c|c|c|c|c|}
\hline & \multicolumn{3}{|c|}{ Liza saliens } & \multicolumn{3}{|c|}{ Liza aurata } & \multicolumn{3}{|c|}{ Liza ramado } \\
\hline & $\chi^{2}$ & g.l. & $\mathrm{P}$ & $\chi^{2}$ & g.l. & $\mathrm{P}$ & $\chi^{2}$ & g.l. & $\mathrm{P}$ \\
\hline Detritus & 8.859 & 3 & 0.031 & 29.107 & 3 & 0.001 & 15.725 & 2 & 0.001 \\
\hline Algas filamentosas & 5.528 & 3 & 0.137 & 2.127 & 3 & 0.547 & 0.806 & 2 & 0.668 \\
\hline Microalgas & 6.427 & 3 & 0.093 & 12.484 & 3 & 0.006 & 7.723 & 2 & 0.021 \\
\hline Arena & 6.092 & 3 & 0.107 & 59.094 & 3 & 0.001 & 11.208 & 2 & 0.004 \\
\hline Insectos & 7.065 & 3 & 0.07 & 6.472 & 3 & 0.091 & 3.636 & 2 & 0.162 \\
\hline Crustáceos & 17.703 & 3 & 0.001 & 25.319 & 3 & 0.001 & 26.477 & 2 & 0.001 \\
\hline Nemátodos & 3.401 & 3 & 0.334 & 21.388 & 3 & 0.001 & 4.719 & 2 & 0.094 \\
\hline Poliquetos & - & - & - & 12.484 & 3 & 0.006 & - & - & - \\
\hline Huevos & 19.997 & 3 & 0.001 & 25.111 & 3 & 0.001 & 35.451 & 2 & 0.001 \\
\hline
\end{tabular}


Tabla 5. Coeficientes de la correlación de Spearman entre la Ls de los estados juveniles del género Liza y la presencia de las distintas presas $(*): \mathrm{P}<0.01$. Spearman correlation coefficients for comparisons between Ls of juvenile stages of Liza species and prey occurrence $(*): P<0.01)$.

\begin{tabular}{|c|c|c|c|c|c|c|c|c|c|c|}
\hline & $L_{s}$ & Detritus & $\begin{array}{c}\text { Algas } \\
\text { filamentosas }\end{array}$ & Microalgas & Arena & Insectos & Crustáceos & Nemátodos & Poliquetos & Huevos \\
\hline$L_{s}$ & 1 & & & & & & & & & \\
\hline Detritus & $0.54^{*}$ & 1 & & & & & & & & \\
\hline Algas filamentosas & 0.06 & 0.01 & 1 & & & & & & & \\
\hline Microalgas & 0.03 & -0.07 & -0.07 & 1 & & & & & & \\
\hline Arena & $0.51^{*}$ & $0.52^{*}$ & $-0.09^{*}$ & 0.01 & 1 & & & & & \\
\hline Insectos & 0.01 & $-0.24^{*}$ & 0.01 & $-0.14^{*}$ & $-0.16^{*}$ & 1 & & & & \\
\hline Crustáceos & $-0.44^{*}$ & $-0.53^{*}$ & -0.03 & $-0.19^{*}$ & $-0.49^{*}$ & -0.07 & 1 & & & \\
\hline Nemátodos & $-0.15^{*}$ & $-0.18^{*}$ & -0.05 & 0.02 & -0.01 & -0.08 & -0.01 & 1 & & \\
\hline Poliquetos & -0.01 & -0.07 & -0.02 & -0.04 & -0.06 & -0.03 & -0.04 & -0.02 & 1 & \\
\hline Huevos & $-0.44^{*}$ & -0.33 & -0.07 & -0.01 & $-0.40^{*}$ & -0.16 & $0.23^{*}$ & 0.07 & -0.06 & 1 \\
\hline
\end{tabular}

relación negativa entre dicha Ls y la presencia de crustáceos y huevos.

\section{DISCUSIÓN}

En conjunto, la dieta de los estados juveniles de mugílidos capturados en la laguna del Mar Menor es omnívora, y está basada principalmente en el consumo de crustáceos planctónicos (Copépodos, Ostrácodos y Misidáceos). Esta situación es similar a la de otras lagunas costeras y estuarios, donde los crustáceos planctónicos conforman el principal componente de la dieta de los juveniles de mugílidos (Ferrari \& Chieregato, 1981; Arias \& Drake, 1990; Gisbert et al., 1996; Baldó \& Drake, 2002).

En los ejemplares estudiados es destacable el importante papel que juegan los detritus y los huevos en la alimentación de las tres especies. En relación con los detritus, estudios realizados en otros sistemas estuáricos y lagunares (lagunas del delta del río Po y del delta del río Ebro, marismas de la bahía de Cádiz y estuario del Guadalquivir) no detectan una frecuencia de aparición tan elevada de este tipo de alimento para las especies objeto de estudio (Ferrari \& Chieregato, 1981; Arias \& Drake, 1990; Gisbert et al., 1996; Baldó \& Drake, 2002). Probablemente, esta situación se debe a que el Mar Menor presenta una elevada productividad de macrófitos bentónicos
(Cymodocea nodosa y Caulerpa prolifera, principalmente) frente a una baja densidad de fitoplancton (Ros \& Miracle, 1984), por lo que hay una mayor disponibilidad de restos de origen vegetal. Con respecto a los huevos (no identificados), la laguna del Mar Menor presenta densidades elevadas y constantes a lo largo del año, conformando parte del componente planctónico (Gilabert, 2001a), recurso que ha sido explotado intensamente por los juveniles de mugílidos durante las cuatro campañas de muestreo.

Los resultados obtenidos destacan la importancia de las microalgas en la dieta de $L$. ramado. Entre otras, destacarían las algas diatomeas y pirrófitas. Esta situación se observa tambien en las marismas de la bahía de Cádiz (Arias \& Drake, 1990) y en las lagunas del delta del río Po (Ferrari \& Chieregato, 1981). Para los juveniles de la especie L. aurata, los insectos (adultos principalmente) suponen una importante fuente de alimento complementaria. Estos resultados concuerdan con los datos obtenidos por Ferrari \& Chieregato (1981) y Gisbert et al. (1996), quienes observan para los juveniles de esta especie, además de para $L$. ramado y L. saliens, una presencia importante de este tipo de presas en sus estómagos.

Con respecto a la variación estacional en el consumo de crustáceos, los juveniles de mugílidos de las tres especies presentaron una menor ocurrencia de este tipo de presas durante la cam- 
paña de verano. Probablemente sea debido a una menor disponibilidad de los mismos durante los meses más cálidos, aspecto que concuerda con los datos de densidad expuestos por Gilabert (2001a).

Las tres especies presentaron un consumo significativamente mayor de detritus durante la campaña de verano, situación que puede estar relacionada con el incremento de la producción del macrofitobentos en la laguna durante esta estación del año y, consecuentemente, de sus restos (detritus). Cabe destacar la correlación positiva entre la Ls de los ejemplares y la presencia de detritus en sus estómagos. Drake \& Arias (1984) asocian este cambio hacia una dieta mas detritívora, a modificaciones en el aparato bucal y digestivo de los peces de mayor talla (disminución de la dentición, engrosamiento de las paredes musculares del estómago y alargamiento del intestino).

Resulta interesante la correlación positiva existente entre la ocurrencia de detritus y de arena en los digestivos, situación debida a la costumbre de estas especies de tomar el alimento filtrando el sustrato, para extraer de él los restos vegetales (Fernández-Delgado et al., 2000).

En cuanto a las diferencias estacionales en la ocurrencia de huevos, se observa como, para las tres especies, hay un mayor consumo de este tipo de presas en las campañas dónde la Ls media de los juveniles es menor. Así, se establece una correlación negativa entre la Ls de los ejemplares y la presencia de huevos en los digestivos. La justificación para esta situación es que los huevos presentan un menor tamaño que el resto de presas potenciales (crustáceos, insectos, etc.) (Gilabert, 2001b), y por lo tanto son consumidas con mayor frecuencia por los individuos más pequeños.

Estas diferencias temporales pueden ser debidas a cambios ontogénicos en la dieta de los mugílidos, circunstancia frecuente en los peces, debido a que muchas especies experimentan cambios en su dieta a medida que van creciendo (Wootton, 1998). En los mugílidos, se han observado cambios en la composición de la dieta en función de la talla de los ejemplares (Ferrari \& Chieregato, 1981; Drake \& Arias, 1984; Baldó \& Drake, 2002), aunque esta circunstancia también puede estar ligada a la variabilidad temporal y espacial en la disponibilidad de los distintos tipos de presas, situación habitual en ambientes lagunares y estuáricos de zonas templadas (Moreira et al., 1992).

En conclusión, la composición de la dieta de los juveniles de mugílidos, en la laguna costera del Mar Menor, presenta diferencias interespecíficas, e intraespecíficas estacionales. En el caso de estas últimas, parece existir un solapamiento entre los cambios ontogénicos en la dieta que ocurren en estas especies y la mayor o menor disponibilidad temporal de las distintas presas.

\section{AGRADECIMIENTOS}

Agradecemos a los miembros de la Línea de Investigación Conservación de Vertebrados Acuáticos del Departamento de Zoología de la Universidad de Murcia su ayuda durante los trabajos de campo. Asimismo, agradecemos al Dr. Javier Gilabert, de la Universidad Politécnica de Cartagena, a José Pedro Marín, del Departamento de Botánica de la Universidad de Murcia, y a María del Mar Sánchez y Jhohi Ismael Quispe, del Departamento de Ecología de la Universidad de Murcia, la ayuda prestada en la identificación de los contenidos estomacales. Parte de este estudio fue financiado por la Consejería de Industria y Medio Ambiente de la Región de Murcia.

\section{BIBLIOGRAFÍA}

ARIAS, A. M. \& P. DRAKE. 1990. Estados alevines y juveniles de la ictiofauna en los caños de las salinas de la bahía de Cádiz. Instituto de Ciencias Marinas de Andalucía. CSIC, Cádiz. 163 pp.

ALMEIDA, P. R. 2003. Feeding ecology of Liza ramada (Risso, 1810) (Pisces, Mugilidae) in a south-western estuary of Portugal. Estuarine, Coastal and Shelf Science, 57: 313-323.

ANDREU-SOLER, A., F. J. OLIVA-PATERNA, C. FERNÁNDEZ-DELGADO \& M. TORRALVA. 2003. Age and growth of the sand smelt, Atherina boyeri (Risso 1810), in the Mar Menor coastal lagoon (SE Iberian Peninsula). Journal of Applied Ichthyology, 19 (4): 202-208. 
BALDÓ, F. \& P. DRAKE. 2002. A multivariate approach to the feeding habits of small fishes in the Guadalquivir Estuary. Journal of Fish Biology, 61 (Supplement A): 21-32.

CARDONA, L. \& F. CASTELLÓ-ORVAY. 1993. Weight loss of starved thick-lipped grey mullet (Chelon labrosus) yearlings at different salinities. Rivista Italiana di Acquacoltura, 28: 13-16.

CARDONA, L. 2001. Non-competitive coexistence between Mediterranean grey mullet: evidence from seasonal changes in food availability, niche breadth and trophic overlap. Journal of Fish Biology, 59: 729-744.

DRAKE, P., A. M. ARIAS \& L. GÁLLEGO. 1984. Biología de los Mugílidos (Osteichthyes, Mugilidae) en los esteros de las salinas de San Fernando (Cádiz). III. Hábitos alimentarios y su relación con la morfometría del aparato digestivo. Investigaciones Pesqueras, 48 (2): 337-367.

FERNÁNDEZ-DELGADO, C., P. DRAKE, A. M. ARIAS \& D. GARCIA. 2000. Peces de Doñana y su entorno. Organismo Autónomo de Parques Nacionales. Ministerio de Medio Ambiente, Madrid. 272 pp.

FERRARI, I. \& A. R. CHIEREGATO. 1981. Feeding habits of juvenile stages of Sparus auratus L., Dicentrarchus labrax L. and Mugilidae in a brackish embayment of the Po River delta. Aquaculture, 25: 243-257.

GILABERT, J. 2001a. Seasonal plankton dynamics in a Mediterranean hypersaline coastal lagoon: the Mar Menor. J.Plankton Res., 23(2): 207-217.

GILABERT, J. 2001b. Short-term variability of the planktonic size structure in a Mediterranean coastal lagoon. J.Plankton Res., 23 (2): 219-226.

GISBERT, E., L. CARDONA \& F. CASTELLÓ. 1996. Resource partitioning among planktivorous fish larvae and fry in a Mediterranean coastal lagoon. Estuarine, Coastal and Shelf Science, 43: 723-735.
GRANADO-LORENCIO, C. 1996. Ecología de peces. Sevilla: Secretariado de Publicaciones. Universidad de Sevilla. 353 pp.

HOTOS, G. N. \& N. VLAHOS. 1998. Salinity tolerance of Mugil cephalus and Chelon labrosus (Pisces: Mugilidae) fry in experimental conditions. Aquaculture, 167: 329-338.

KOUTRAKIS, E. T., C. T. ATHANASSIOS \& I. S. APOSTOLOS. 2005. Temporal variability of the ictiofauna in a Northern Aegean coastal lagoon (Greece). Influence of environmental factors. Hydrobiologia, 543: 245-257.

LAFFAILLE, P., E. FEUNTEUN, C. LEFEBVRE, A. RADREAU, G. SAGAN \& J. C. LEFEUVRE. 2002. Can thin-lipped mullet directly exploit the primary and detritic production of european macrotidal salt marshes? Estuarine, Coastal and Shelf Science, 54: 729-736.

MOREIRA, F., C. A. ASSIS, P. R. ALMEIDA, J. L. COSTA \& M. J. COSTA. 1992. Trophic relationships in the community of the upper Tagus Estuary (Portugal): A preliminary approach. Estuarine, Coastal and Shelf Science, 34: 617-623.

PÉREZ-RUZAFA, A. \& C. MARCOS DIEGO. 2003. El Mar Menor. In: Los recursos naturales de la Región de Murcia. Un análisis interdisciplinar. M. A. Esteve, M. Lloréns \& C. Martínez (eds.): 404-411. Universidad de Murcia, Servicio de Publicaciones. Murcia. España.

ROS, M. \& M. R. MIRACLE. 1984. Distribución temporal de las dinoflageladas del Mar Menor. Anales de Biología, 2: 169-180.

STRYDOM, N. A. 2003. Occurrence of larval and early juveniles fishes in the surf zone adjacent to two intermittently open estuaries, South Africa. Environmental Biology of Fishes, 66: 349-359.

WOOTTON, R. J. 1998. Ecology of teleost fishes. Kluwer Academic Publishers, Dordrecht. 386 pp. 
